Article

\title{
Effects of Respiratory Muscle Warm-up on High-Intensity Exercise Performance
}

\author{
Taylor S. Thurston, Jared W. Coburn, Lee E. Brown, Albert Bartolini, Tori L. Beaudette, \\ Patrick Karg, Kathryn A. McLeland, Jose A. Arevalo, Daniel A. Judelson and Andrew J. Galpin * \\ Center for Sport Performance, Department of Kinesiology, California State University, Fullerton, \\ CA 92834, USA; E-Mails: taylor.thurston@utah.edu (T.S.T.); Jcoburn@fullerton.edu (J.W.C.); \\ leebrown@fullerton.edu (L.E.B.); abartolini@fullerton.edu (A.B.); TBeaudette@csusb.edu (T.L.B.); \\ pkarg@fullerton.edu (P.K.); k.a.mcleland@gmail.com (K.A.M.); jarevalo@fullerton.edu (J.A.A.); \\ dan.judelson@nike.com (D.A.J.)
}

* Author to whom correspondence should be addressed; E-Mail: agalpin@fullerton.edu; Tel.: +1-657-278-2112; Fax: +1-657-278-5317.

Academic Editor: Eling de Bruin

Received: 21 September 2015 / Accepted: 30 October 2015 / Published: 5 November 2015

\begin{abstract}
Exercise performance is partially limited by the functionality of the respiratory musculature. Training these muscles improves steady-state exercise performance. However, less is known about the efficacy of executing a respiratory muscle warm-up (RWU) immediately prior to high-intensity exercise. Our study purpose was to use a practitioner-friendly airflow restriction device to investigate the effects of a high, medium, or low intensity RWU on short, high-intensity exercise and pulmonary, cardiovascular, and metabolic function. Eleven recreationally active, males $(24.9 \pm 4.2 \mathrm{y}, 178.8 \pm 9.0 \mathrm{~cm}, 78.5 \pm 10.4 \mathrm{~kg}, 13.4 \% \pm 4.2 \%$ body fat) cycled at $85 \%$ peak power to exhaustion (TTE) following four different RWU conditions (separate days, in random order): (1) high; (2) medium; (3) low airflow inspiration restriction, or no RWU. When analyzed as a group, TTE did not improve following any RWU (4.73 $\pm 0.33 \mathrm{~min})$. However, 10 of the 11 participants improved $\geq 25 \mathrm{~s}$ in one of the three RWU conditions (average $=47.6 \pm 13.2 \mathrm{~s}$ ), which was significantly better than $(p<0.05)$ the control trial (CON). Neither blood lactate nor perceived difficulty was altered by condition. In general, respiratory exchange ratios were significantly lower during the early stages of TTE in all RWU conditions. Our findings suggest RWU efficacy is predicated on identifying optimal inspiration intensity, which clearly differs between individuals.
\end{abstract}


Keywords: intervals; high-intensity; performance; respiratory; hypoxia; breathing; restriction; hypocapnia; fatigue

\section{Introduction}

Numerous physiological factors such as $\mathrm{pH}$, temperature, neurological input, and substrate availability limit exercise performance. One particularly understudied aspect is the role of the respiratory musculature. The available literature provides clear evidence that fatigue of this system hampers exercise performance [1-4] by inducing a metaboreflex response, which causes sympathetic outflow and a resulting vasoconstriction/reduced oxygen delivery to working muscles [3,5-9]. Fortunately, functional capacity of the respiratory musculature is modifiable. Intentionally restricting airflow during inhalation, exhalation, or both [10-12] forces the respiratory muscles to increase work output to adequately support airflow and gas exchange needs. Improvements in whole-body performance have been found in a variety of sports [12-15] and general exercise settings [16-18] following these types of respiratory exercise. For example, Romer, McConnell, and Jones (2002) reported that chronic respiratory training significantly improved $20 \mathrm{~km}$ and $40 \mathrm{~km}$ cycling time trial performance by 3\%-5\% [12].

Restrictive airflow breathing activities may also improve acute exercise performance by functioning as a pre-exercise respiratory muscle warm-up (RWU). This is of particular importance when engaging in high-intensity exercise. The respiratory muscles have high aerobic capacities, multiple sources of blood supply, and a unique resilience to vasoconstriction; making them naturally fatigue-resistant to low-moderate exercise intensities. However, they are susceptible to fatigue when exercising at intensities greater than $80 \%$ of $\mathrm{VO}_{2} \max$ for prolonged periods of time [5]. Volianitis and colleagues (1999) understood this phenomenon and sought to examine whether or not a RWU (which presumably improves function of the respiratory muscles) could sufficiently alter high intensity exercise performance.

This was accomplished by comparing performance during a six-minute maximal rowing test following either a sport-specific warm-up or a RWU. The researchers noted the RWU induced a significant increase in rowing distance of $18 \mathrm{~m}$. On the contrary, Johnson et al., (2014) found no change in $10 \mathrm{~km}$ cycling time trial performance following a RWU combined with a sport specific warm-up (compared to a sport specific warm-up alone). Thus, while RWU appears helpful when utilized prior to short duration, high-intensity rowing, its effectiveness may be eliminated when cycling. Further examination of this speculation is important given the popularity of high-intensity cycling among personal trainers, strength and conditioning coaches, and health professionals.

Confusing the matter even more, the intensity (i.e., amount of airflow restriction) and volume of the RWU significantly affect its outcome. Most studies report success when implementing two sets of 30 respiratory cycles at $40 \%$ of maximal inspiratory pressure [1-15,18]. Higher intensity restriction actually fatigues the system, while less restriction is insufficient [19]. Measurement of maximal inspiratory pressure typically requires specialized, expensive equipment. Practitioners attempting to implement a RWU are unlikely to possess such devices. However, less expensive RWU devices that allow manipulation of airflow restriction intensity are commercially available, but lack scientific scrutiny. Therefore, the purpose of this study was to utilize one of these practitioner friendly devices to 
investigate the effects of a high, medium, or low intensity RWU on cycling time to exhaustion and pulmonary, cardiovascular, and metabolic function.

\section{Materials and Method}

\subsection{Study Design}

This study was designed to investigate the effectiveness of three different RWU intensities on exercise performance. Day 1 consisted of baseline measurements and familiarization of the RWU protocol. On Days 2-5, participants performed a RWU with either high (HI), medium (MID), or low (LO) amounts of airflow restriction (or no RWU CON) immediately prior to a cycling test to exhaustion (TTE). Each trial (HI, MID, LO, CON) was separated by at least $48 \mathrm{~h}$, and the order of each trial was randomized. Exercise performance and measures of pulmonary, cardiovascular, and metabolic function were collected and analyzed during each of the four trials.

\subsection{Study Controls}

Trial order was randomized via a Latin Squares design, with each trial occurring at least 48, but no more than 96, h apart. All trials occurred in the morning between 6:00 and 10:00 am, with each trial occurring $\pm 1 \mathrm{~h}$ from the first experimental visit for each individual participant. To ensure training status did not change throughout the study, participants kept a training log for the week prior to their first testing session. Activity levels were then mimicked and recorded their throughout the duration of their involvement in the study. Participants were asked to refrain from exercise for the $36 \mathrm{~h}$ before testing and were encouraged to sleep $\geq 8 \mathrm{~h}$ the night before each test. Similar measures were taken for dietary intake during the $24 \mathrm{~h}$ period that preceded each individual testing session. Participants fasted $12 \mathrm{~h}$ prior to each testing session. Nonetheless, three participants were removed from the study because of significant differences in their carbohydrate intake the $24 \mathrm{~h}$ prior to one or more of their trials. To ensure sufficient hydration, participants were instructed to drink water the night before $(\sim 1000 \mathrm{~mL})$ and the morning of ( $\sim 500 \mathrm{~mL}$ ) testing. Hydration status was monitored via urine specific gravity analysis prior to each trial. Recordings of greater than 1.020 resulted in a rescheduling of the exercise trial [20]. No external motivation (e.g., cheering, yelling, music, etc.) was provided or allowed during the RWU, pulmonary testing, or the TTE.

\subsection{Participants}

Fourteen healthy male recreational exercisers enrolled in the study, but three were removed from the final analyses ( $n=11$, Table 1). Participants were excluded prior to beginning any activities of the study if they had any prior experience with RWU (or similar) practices. Inclusion criteria required participants be free from any cardiovascular, metabolic, and pulmonary diseases or illnesses that may affect the pulmonary system, or have any joint, musculoskeletal, and/or neuromuscular injuries or pain that may affect their ability to perform maximal cycling exercise. To ensure adequate levels of aerobic fitness, participants were also required to demonstrate a $\mathrm{VO}_{2} \max$ of $\geq 45 \mathrm{~mL} \cdot \mathrm{kg}^{1} \cdot \mathrm{min}^{-1}$. Prior to participating, all volunteers were informed of the risks and signed a document of informed consent. This document, as well as the study, were approved by The University Institutional Review Board. 
Table 1. Participant descriptive information.

\begin{tabular}{cc}
\hline Variable & Mean \pm SD \\
\hline Age $(\mathrm{y})$ & $24.9 \pm 4.2$ \\
Height $(\mathrm{cm})$ & $178.8 \pm 9.0$ \\
Mass $(\mathrm{kg})$ & $78.5 \pm 10.4$ \\
Body Fat $(\%)$ & $13.4 \pm 4.2$ \\
$\mathrm{VO}_{2 \max }\left(\mathrm{mL} \cdot \mathrm{kg}^{-1} \cdot \mathrm{min}^{-1}\right)$ & $54.8 \pm 6.9$ \\
Cycling Intensity $(\mathrm{W})$ & $274.5 \pm 45.8$ \\
\hline
\end{tabular}

\subsection{Preliminary Testing}

During Visit 1, investigators assessed height and mass via stadiometer (SECA, Ontario, CA, USA) and an electronic scale (Healthometer Toledo ES200L, Ohaus, Pine Brook, NJ, USA), respectively. Body fat percentage was calculated via a three site skinfold protocol from the chest, thigh, and abdomen [21]. Participants then performed a cycle ergometer (Ergomedic 839E, Monarch, Stockholm, Sweden) $\mathrm{VO}_{2}$ max test. Expired gasses were measured using a TRUEMAX 2400 metabolic cart (PARVOMEDICS, Sandy, UT, USA), calibrated before each trial. The test required participants to initially cycle at $50 \mathrm{~W}$ for five minutes. The test subsequently increased $25 \mathrm{~W}$ every minute until the participant was too exhausted to continue, $\mathrm{VO}_{2}$ plateaued, heart rate (HR) (Polar Electro Inc., FS1 and TS1, Woodbury, NY, USA) failed to increase despite increasing intensity, a respiratory exchange ratio (RER) greater than 1.15 was achieved, or the participant indicated a rating of perceived exertion (RPE) of greater than 18 [22]. Following the $\mathrm{VO}_{2}$ max test, participants were familiarized with the RWU protocol. This consisted of 2 sets of 30 breaths ( 1 min rest between sets) [19] while the nose was clipped shut and an air flow inhalation restriction device $\left(\mathrm{O}_{2}\right.$ Trainer ${ }^{\mathrm{TM}}$, Westwood, CA, USA) was placed in the mouth. The device (Figure 1) was set to the highest restriction setting possible during the familiarization for all participants to ensure they were capable of completing the protocol during HI. Participants were instructed to both inhale and exhale with maximum effort per breath.

\subsection{Experimental Trials}

Visit 2-5 consisted of either a RWU with HI, MID, or LO airflow restriction, or no RWU of any type (CON), followed by TTE. RWU was accomplished by performing 2 sets of 30 repetitions (1 min rest between sets) of maximal breaths at their normal breathing cadence. During CON, participants rested quietly on the cycle and performed no respiratory warm-up. The time between the end of the warm-up and the start of TTE was matched between all four experimental conditions. Participants used the same device for all familiarization and experimental trials. The specific RWU volumes and intra-set rest intervals used here replicated procedure used in previous studies [13-18]. The device was specifically chosen because it is inexpensive, portable, and allows the amount of airflow restriction to be adjusted (range of 3-13 mm), making it a realistic option for practitioners. Moreover, the device includes a three way valve built into the mouthpiece allowing airflow to be restricted during inhalation, yet unrestricted during exhalation. To contribute to this literature regarding optimal inspiratory intensity, we included RWU conditions across the spectrum of airflow inhalation restriction allowed by the device. Linear changes in airflow restriction diameter settings on the device likely translate into exponential changes in flow rate 
and/or inspiratory pressure. Thus, we chose to perform HI with a $3 \mathrm{~mm}$ opening (fitting \#13), MID with an $8 \mathrm{~mm}$ opening (fitting \#7), and LO with a $13 \mathrm{~mm}$ opening (fitting \#2).

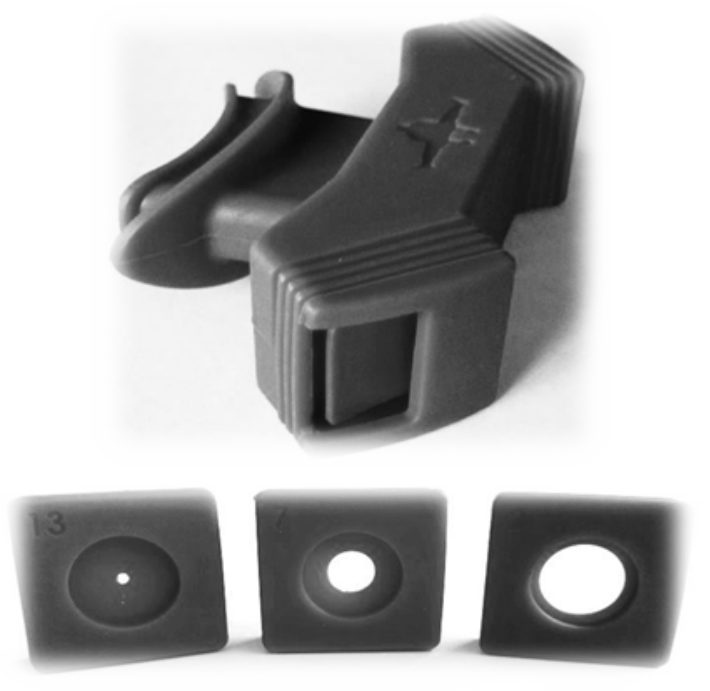

Figure 1. Photo of airflow restriction device used for the respiratory warm-up. Interchangeable caps are placed on one side of the device, restricting inhalation of air to the desired diameter, but allowing unrestricted exhalation. The high restriction (HI) warm-up used the $3 \mathrm{~mm}$ opening (fitting \#13), the medium restriction (MID) used the $8 \mathrm{~mm}$ opening (fitting \#7), and the low restriction (LO) used the $13 \mathrm{~mm}$ opening (fitting \#2).

Upon arrival at the laboratory for all experimental trials, participants were assessed for hydration and training/nutrition logs were examined. After verifying compliance with all pre-study controls, participants were fitted to the cycle ergometer and the metabolic mask was sized to the participant's head and mouth. Personal cycle settings were recorded and reproduced for subsequent trials. After a five minute rest, blood lactate was measured via finger prick of the index finger (Accutrend Lactate Analyzer, Roche Diagnostics, Basel, Switzerland). Participants then performed pulmonary function testing via spirometry (SpiroLab II, SDI Diagnostics, Easton, MA, USA) until three repeatable values were recorded.

Volunteers then performed a five minute warm-up at $50 \mathrm{~W}$ on the cycle ergometer. Then, while remaining on the cycle, they performed the appropriate RWU protocol. Another pulmonary function assessment was conducted following the completion of the last set of RWU. This testing, and all pre-TTE preparations (e.g., mask set-up, etc.) were completed within two minutes of completing the cycling warm-up. TTE was performed at $85 \%$ of the participants adjusted peak wattage (achieved during the $\mathrm{VO}_{2}$ max test). RPE was measured at 1 min intervals using the Borg 6-20 scale. Volume of oxygen exhaled $\left(\mathrm{VO}_{2}\right)$, volume of carbon dioxide exhaled $\left(\mathrm{VCO}_{2}\right)$, RER, minute ventilation (VE), and HR were recorded using $15 \mathrm{~s}$ averages during the TTE. The TTE was terminated when the exerciser failed to maintain $\geq 60$ revolutions per minute for five consecutive seconds [23,24]. Blood lactate was taken immediately after TTE was terminated. Five minutes of rest on the ergometer was given to participants before a post-exercise pulmonary function test was completed. 


\subsection{Statistical Analyses}

Performance during TTE was analyzed by using a 4 (RWU Intensity) $\times 1$ (time) repeated measures analysis of variance (ANOVA). A 4 (RWU Intensity) $\times 2$ (pre and post TTE) repeated ANOVA was used to assess statistical differences among treatments for blood lactate. A 4 (RWU Intensity) $\times 6$ (pre, $1 \mathrm{~min}, 2 \mathrm{~min}, 3 \mathrm{~min}, 4 \mathrm{~min}$, and post TTE) repeated ANOVA was used to assess statistical differences among treatments for RPE. A $t$-test was used to compare the each individual's best performance against CON. Forced vital capacity (FVC), forced expiratory volume in $1 \mathrm{~s}\left(\mathrm{FEV}_{1}\right)$, forced expiratory volume percentage (FEV1\%), forced inspiratory vital capacity (FIVC), and peak expiratory flow (PEF) were analyzed via separate 4 (RWU Intensity) $\times 3$ (baseline, post RWU/pre TTE, and post TTE) repeated ANOVA. Metabolic data were analyzed only to the point of the earliest TTE. Thus, 4 (RWU Intensity) $\times 13$ (time) repeated ANOVA were used to assess statistical differences among treatments for absolute $\mathrm{VO}_{2}, \mathrm{VCO}_{2}, \mathrm{RER}, \mathrm{VE}$, and $\mathrm{HR}$. In the presence of a significant $\mathrm{F}$ ratio, Tukey's post hoc test was used to analyze pair-wise comparisons. The alpha level was set at 0.05 .

\section{Results}

\subsection{Time to Exhaustion}

There were no significant differences $(p \geq 0.05)$ across conditions for TTE $(4.73 \pm 0.33 \mathrm{~min})$. However, 10 of the 11 participants displayed a noticeable improvement ( $\geq 25 \mathrm{~s})$ in one of the three experimental conditions (HI, MID, or LO) when compared to CON. Five had their best performance during MID (Figure 2), three during LO (Figure 3), and two during HI (Figure 4). The average improvement among these best trials was $47.6 \pm 13.2 \mathrm{~s}$, which was significantly different than CON.

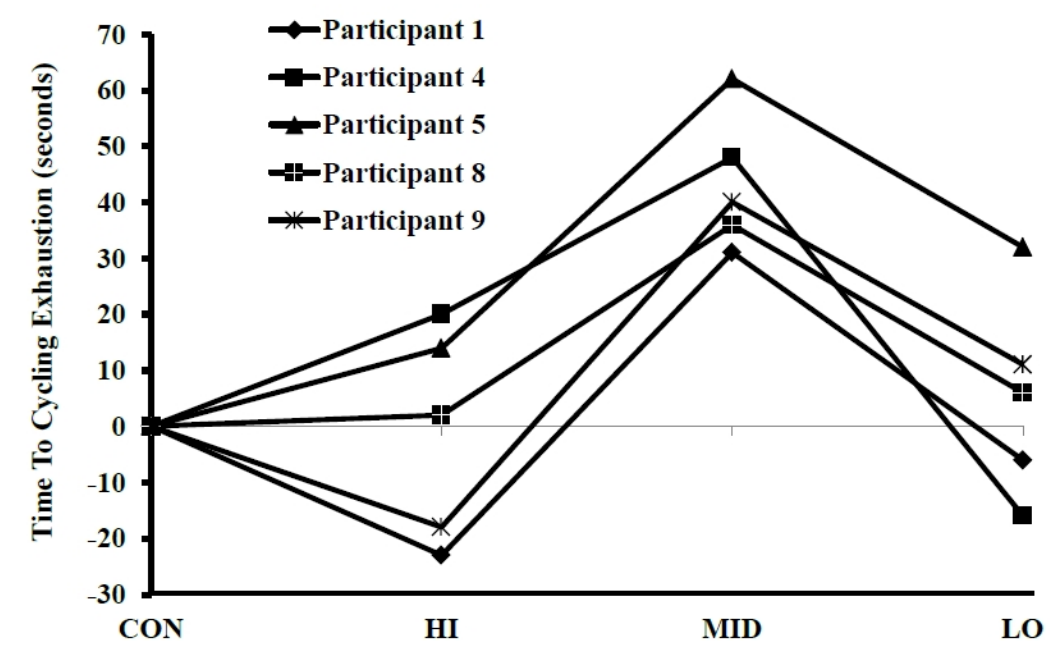

Figure 2. Changes in time to exhaustion during a cycling test immediately following a respiratory warm-up, which consisted of two sets of 30 repeitions of maximal breathes while wearing an air flow inhalation restriction device set at $13 \mathrm{~mm}$ (HI), $8 \mathrm{~mm}$ (MID), or $3 \mathrm{~mm}$ (LO) of restriction. A control trial (CON) was performed with no warm-up. Performance during CON was considered zero. The amount of time (seconds) more or less than CON is plotted above for each participant who experienced their best perfomrance during MID. 


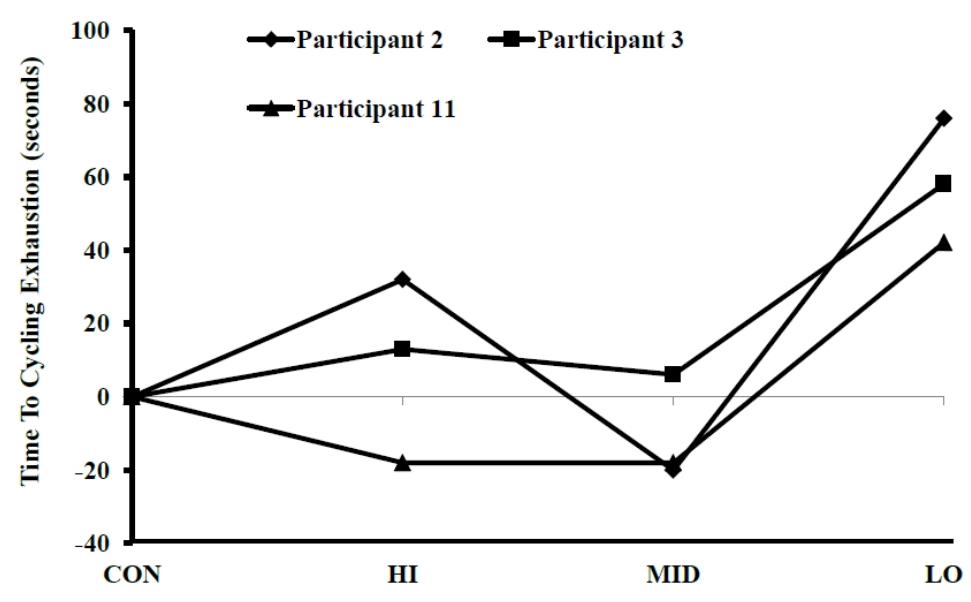

Figure 3. Changes in time to exhaustion during a cycling test immediately following a respiratory warm-up, which consisted of two sets of 30 repeitions of maximal breathes while wearing an air flow inhalation restriction device set at $13 \mathrm{~mm}$ (HI), $8 \mathrm{~mm}$ (MID), or $3 \mathrm{~mm}$ (LO) of restriction. A control trial (CON) was performed with no warm-up. Performance during CON was considered zero. The amount of time (seconds) more or less than CON is plotted above for each participant who experienced their best perfomrance during LO.

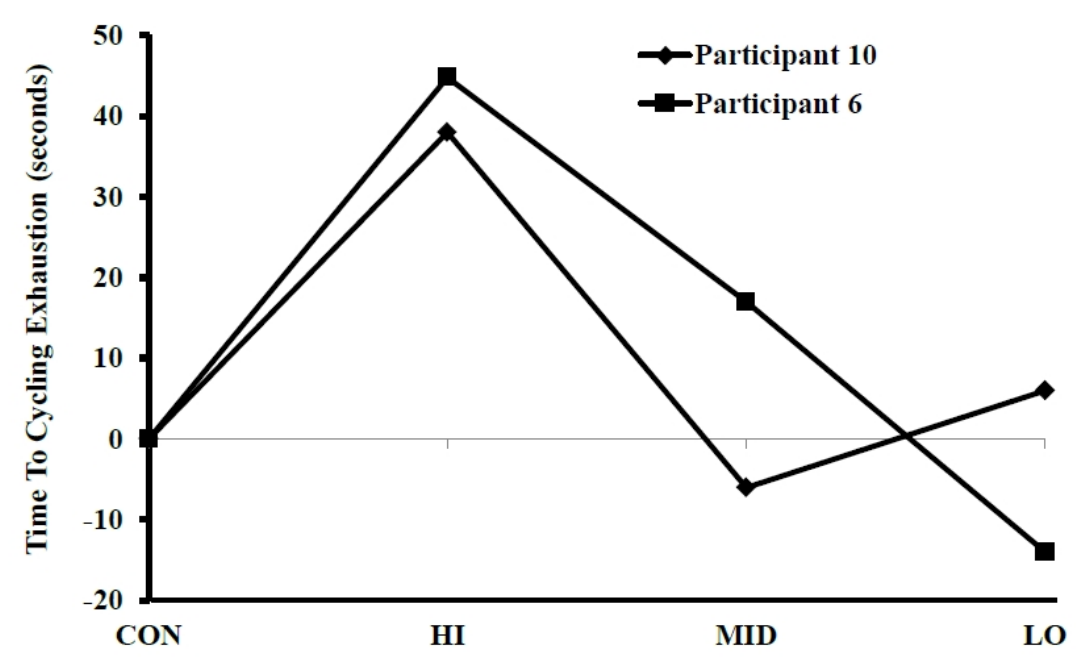

Figure 4. Changes in time to exhaustion during a cycling test immediately following a respiratory warm-up, which consisted of two sets of 30 repeitions of maximal breathes while wearing an air flow inhalation restriction device set at $3 \mathrm{~mm}$ (LO), $8 \mathrm{~mm}$ (MID, or $13 \mathrm{~mm}$ (HI) of restriction. A control trial (CON) was performed with no warm-up. Performance during CON was considered zero. The amount of time (seconds) more or less than CON is plotted above for each participant who experienced their best perfomrance during HI.

\subsection{Rating of Perceived Exertion and Lactate}

There were significant main effects, but not condition interactions, of time for lactate (Table 2) or RPE (Pre $=7.8 \pm 1.4$, Post $=20.0 \pm 0.0$ ). 
Table 2. Lactate before and after a time to exhaustion trial. Data are reported as mean \pm SD.

\begin{tabular}{ccc}
\hline Condition & Pre & Post \\
\hline HI & $1.95 \pm 0.56$ & $15.38 \pm 4.49$ \\
MID & $1.91 \pm 0.40$ & $15.80 \pm 4.03$ \\
LOW & $2.17 \pm 0.82$ & $13.74 \pm 4.14$ \\
CON & $1.88 \pm 0.47$ & $14.20 \pm 3.70$ \\
\hline
\end{tabular}

\subsection{Pulmonary Function}

No time main effects, condition main effects, or time by condition interactions were reported for $\mathrm{FVC}, \mathrm{FEV}_{1}, \mathrm{FEV}_{1} \%$, or FIVC. A significant main effect of time and an interaction by condition existed for PEF. Baseline (10.33 $\pm 1.54,10.33 \pm 1.48,10.33 \pm 1.49 \mathrm{~L} / \mathrm{s})$ and post TTE $(10.26 \pm 1.47$, $10.36 \pm 1.70,10.35 \pm 1.61$ ) were not different from each other, but were both significantly greater than post RWU $(9.89 \pm 1.55,9.88 \pm 1.84,9.68 \pm 1.42)$ for HI, MID, and CON, respectively. Baseline PEF $(10.30 \pm 1.68)$ was significantly greater than both post RWU $(9.80 \pm 1.81)$ and post TTE $(10.06 \pm 1.80)$ during LO.

\subsection{Gas Exchange}

A main effect of time was statistically significant for $\mathrm{VCO}_{2}, \mathrm{VO}_{2}$, RER, and $\mathrm{HR}$. However, interaction effects between condition and time were only significant for $\mathrm{VCO}_{2}$, RER, and VE, while a trend ( $p<$ 0.10) existed for $\mathrm{VO}_{2}$. Regarding $\mathrm{VCO} 2$, $\mathrm{CON}$ was significantly greater than MID for all time points up to one minute and $30 \mathrm{~s}$. For time points between $45 \mathrm{~s}$ and one minute and $30 \mathrm{~s}$, CON was significantly greater than LOW. Regarding RER, CON was significantly higher than HI, MID, and LO for all time points up to one minute and $30 \mathrm{~s}$, and higher than MID for all time points up to two minutes and $30 \mathrm{~s}$. For VE, CON was significantly greater than LOW and MID, and HI was significantly greater than MID at the one-minute time point.

\section{Discussion}

The primary findings of this study indicate the effectiveness of the inspiratory RWU is highly contingent upon the intensity (i.e., amount of airflow restriction) being optimized for the individual. If accomplished, the RWU protocol resulted in statistically significant and practically important changes in short-term, cycling to exhaustion performance. Our results also suggest that RWU, regardless of intensity, significantly decreased early stage (of exercise) RER, without altering blood lactate or perceived exertion, or decreasing exercise performance.

\subsection{Performance}

The interpretation that our RWU provided no performance benefit also holds merit as all conditions (HI, MID, and LO) failed to reach statistical significance. Several potential explanations exist. Firstly, the time between our RWU and the start of our exercise differed from some of the previous research. The current study followed the general methods of previous literature with similar procedures $[16,18]$. However, we chose to implement a rest interval that was shorter than previous research as it better 
reflected practices during normal exercise settings. Others have found performance enhancements when engaging in the RWU 30 min before exercise [25,26]. The optimal rest interval between RWU and exercise remains unclear, but our findings, combined with previous research [25,26], suggest longer may be better than shorter. Secondly, the limitations of the RWU device precluded us from controlling and/or quantifying inspiratory pressure or flow rate during the RWU, meaning we could not standardize conditions between or within participants. This directly influenced our grouped performance results.

Most previous research in this area has focused on identifying physiological mechanisms associated with RWU, not necessarily practitioner-friendly applications. This important stage in the research required scientists to control and monitor as many variables as possible [13-18], and led to the prescription of a desired inspiratory pressure of 40\% [19]. Our goal was to examine RWU in setting more realistic to practitioners. Thus, we chose to use an inexpensive, commercially available device, knowing it could not be set to an optimal airflow restriction (per participant) a priori. We directly examined the loading intensity issue by asking participants to repeat the TTE trial under three different settings (which spanned the ability of the device, and was reasonable to complete the protocol). Finding significant differences among conditions was therefore difficult because of the high deviations in performance between each person's trials. This was particularly evident by the participants who had decreases in performance of $\geq 18 \mathrm{~s}$ (when compared to CON) during HI. These findings support previous research and suggest an optimal range exists for the intensity of a RWU, with too low of an intensity failing to produce benefit and too high of an intensity causing premature respiratory muscle fatigue [19]. However, significant improvements $(p<0.05)$ in performance were noted when the best trial per person was compared to their own CON trial. No clear patterns emerged among participants who performed best at a given RWU intensity. In conclusion: (1) an ideal inspiratory pressure exists; (2) previous research shows significant benefits of respiratory training $[13,14,17,18]$; and (3) individualized performance benefits of 10 of the 11 participants were more than double the standard deviation $( \pm 20 \mathrm{~s})$ better than CON during at least one of the RWU conditions. This collective evidence allows us the confidence to conclude the protocol has the potential to provide practically important benefits to the physical performance test. The viability of a RWU under different circumstances deserves further investigation. For example, our RWU protocol only targeted the inspiratory muscles. Protocols which target the expiratory muscles (or both) may yield different findings.

\subsection{Metabolic Function}

The RWU significantly altered ventilation and $\mathrm{O}_{2} / \mathrm{CO}_{2}$, at least during the first two minutes of exercise. Specifically, the decrease in RER appeared to mirror those of $\mathrm{VCO}_{2}$, but over a slightly longer time point. This was matched by a corresponding increase in $\mathrm{VO}_{2}$ (trend). These factors could explain the enhanced VE displayed during the first minute of exercise, and combine to suggest the RWU induced arterial hypocapnia. Unfortunately, this speculation cannot be confirmed as measurement of endtidal $\mathrm{CO}_{2}$ during the RWU was not possible. Previous studies have found hyperventilation-induced hypocapnia increases locomotor vasoconstriction, decreases muscle perfusion, and reduces $\mathrm{O}_{2}$ delivery $[27,28]$, which should combine to produce a diminution of exercise performance. Yet, here we report performance enhancement. Explanation of this apparent conundrum is not directly possible with the given data as specific examination of the interplay between RWU and hypocapnia requires an entirely 
different study design and focus. The authors can only speculate that either hypocapnia did not occur, or the RWU offset any potential detriments.

\subsection{Blood Lactate and Pulmonary Function}

Blood lactate was not different between RWU conditions, meaning it does not explain the changes in performance. Similar conclusions were reported in other related studies [14-16]. As expected, performance enhancements cannot be explained by acute changes in pulmonary function either as it was also generally unresponsive to the RWU, which was expected [4,12,14,29-31]. PEF did show condition specific responses, but the magnitude of change $(<8 \%)$ indicates a limited clinical relevance [32]. Researchers should consider designing future research in a manner that allows specific evaluation of the pulmonary, metabolic, perceptual, muscular, neurological or other mechanisms responsible for performance enhancements.

\section{Conclusions}

The current study provides unique insight into the effects of RWU. Our data indicate RWU may enhance short-term, high-intensity exercise performance, but only when performed at an optimal intensity (i.e., airflow restriction). Practitioners should implement with caution as intensity differs between individuals, and too difficult of a RWU hinders exercise performance. Further examination of the relationship between (1) performance-based classifications of optimal airflow restriction and (2) previously established guidelines of $40 \%$ maximum inspiratory pressure [19] would provide validation to both approaches and afford coaches/users the ability to effectively incorporate RWU into practice. It is unknown if other warm-up protocols (e.g., different volumes, longer rest between RWU, etc.) provide similar benefits. Moreover, the effectiveness of this RWU on exercise of other intensities, durations, or modes (e.g., running, rowing, swimming, etc.) is unknown. Researchers should also apply a similar, application-based research approach to study participants from other populations, particularly those with cardiopulmonary abnormalities. Further investigations in this area would benefit both the scientific community and practitioners.

\section{Acknowledgments}

The research team would like to thank all the participants for their hard work and effort in this exhausting task.

\section{Author Contributions}

Taylor S. Thurston, Jared W. Coburn, Lee E. Brown, Daniel A. Judelson and Andrew J. Galpin conceived and designed the experiments; Taylor S. Thurston, Albert Bartolini, Tori L. Beaudette, Patrick Karg, Kathryn A. McLeland, and Jose A. Arevalo performed the experiments; Taylor S. Thurston, Jared W. Coburn, Lee E. Brown, Daniel A. Judelson, and Andrew J. Galpin analyzed the data; Andrew J. Galpin contributed reagents/materials/analysis tools; Taylor S. Thurston, Jared W. Coburn, Lee E. Brown, Daniel A. Judelson and Andrew J. Galpin wrote the paper. 


\section{Conflicts of Interest}

The authors declare no conflict of interest.

\section{References}

1. Mador, M.J.; Acevedo, F.A. Effect of respiratory muscle fatigue on subsequent exercise performance. J. Appl. Physiol. 1991, 70, 2059-2065.

2. Verges, S.; Sager, Y.; Erni, C.; Spengler, C.M. Expiratory muscle fatigue impairs exercise performance. Eur. J. Appl. Physiol. 2007, 101, 225-232.

3. Romer, L.M.; Polkey, M.I. Exercise-induced respiratory muscle fatigue: Implications for performance. J. Appl. Physiol. 2008, 104, 879-888.

4. Volianitis, S.; McConnell, A.K.; Jones, D.A. Assessment of maximum inspiratory pressure. Prior submaximal respiratory muscle activity (“warm-up”) enhances maximum inspiratory activity and attenuates the learning effect of repeated measurement. Respir. Int. Rev. Thorac. Dis. 2001, 68, 22-27.

5. Dempsey, J.A.; Romer, L.; Rodman, J.; Miller, J.; Smith, C. Consequences of exercise-induced respiratory muscle work. Respir. Physiol. Neurobiol. 2006, 151, 242-250.

6. Hill, J.M. Discharge of group iv phrenic afferent fibers increases during diaphragmatic fatigue. Brain Res. 2000, 856, 240-244.

7. Jammes, Y.; Balzamo, E. Changes in afferent and efferent phrenic activities with electrically induced diaphragmatic fatigue. J. Appl. Physiol. 1992, 73, 894-902.

8. Harms, C.A.; Babcock, M.A.; McClaran, S.R.; Pegelow, D.F.; Nickele, G.A.; Nelson, W.B.; Dempsey, J.A. Respiratory muscle work compromises leg blood flow during maximal exercise. J. Appl. Physiol. 1997, 82, 1573-1583.

9. Sheel, A.W.; Derchak, P.A.; Morgan, B.J.; Pegelow, D.F.; Jacques, A.J.; Dempsey, J.A. Fatiguing inspiratory muscle work causes reflex reduction in resting leg blood flow in humans. J. Physiol. 2001, 537, 277-289.

10. Verges, S.; Lenherr, O.; Haner, A.C.; Schulz, C.; Spengler, C.M. Increased fatigue resistance of respiratory muscles during exercise after respiratory muscle endurance training. Am. J. Physiol. Regul. Integr. Comp. Physiol. 2007, 292, R1246-R1253.

11. Verges, S.; Renggli, A.S.; Notter, D.A.; Spengler, C.M. Effects of different respiratory muscle training regimes on fatigue-related variables during volitional hyperpnoea. Respir. Physiol. Neurobiol. 2009, 169, 282-290.

12. Romer, L.M.; McConnell, A.K.; Jones, D.A. Inspiratory muscle fatigue in trained cyclists: Effects of inspiratory muscle training. Med. Sci. Sports Exerc. 2002, 34, 785-792.

13. Volianitis, S.; McConnell, A.K.; Koutedakis, Y.; Jones, D.A. Specific respiratory warm-up improves rowing performance and exertional dyspnea. Med. Sci. Sports Exerc. 2001, 33, 1189-1193.

14. Wilson, E.E.; McKeever, T.M.; Lobb, C.; Sherriff, T.; Gupta, L.; Hearson, G.; Martin, N.; Lindley, M.R.; Shaw, D.E. Respiratory muscle specific warm-up and elite swimming performance. Br. J. Sports Med. 2013, 48, 789-791. 
15. Lin, H.; Tong, T.K.; Huang, C.; Nie, J.; Lu, K.; Quach, B. Specific inspiratory muscle warm-up enhances badminton footwork performance. Appl. Physiol. Nutr. Metab. Physiol. Appl. Nutr. Metab. 2007, 32, 1082-1088.

16. Cheng, C.F.; Tong, T.K.; Kuo, Y.C.; Chen, P.H.; Huang, H.W.; Lee, C.L. Inspiratory muscle warm-up attenuates muscle deoxygenation during cycling exercise in women athletes. Respir. Physiol. Neurobiol. 2013, 186, 296-302.

17. Tong, T.K.; Fu, F.H. Effect of specific inspiratory muscle warm-up on intense intermittent run to exhaustion. Eur. J. Appl. Physiol. 2006, 97, 673-680.

18. Lomax, M.; Grant, I.; Corbett, J. Inspiratory muscle warm-up and inspiratory muscle training: Separate and combined effects on intermittent running to exhaustion. J. Sports Sci. 2011, 29, 563-569.

19. Roussos, C.S.; Macklem, P.T. Diaphragmatic fatigue in man. J. Appl. Physiol. Respir. Environ. Exerc. Physiol. 1977, 43, 189-197.

20. Armstrong, L.E.; Maresh, C.M.; Castellani, J.W.; Bergeron, M.F.; Kenefick, R.W.; LaGasse, K.E.; Riebe, D. Urinary indices of hydration status. Int. J. Sport Nutr. 1994, 4, 265-279.

21. Jackson, A.S.; Pollock, M.L. Practical assessment of body composition. Phys. Sports Med. 1985, 13, 76-90.

22. American College of Sports Medicine. Acsm's Guidelines for Exercise Testing and Prescription, 7th ed.; Lippincott Williams \& Wilkins: Philadelphia, PA, USA, 2006; p. 366.

23. Ghosh, A.K.; Rahaman, A.A.; Singh, R. Combination of sago and soy-protein supplementation during endurance cycling exercise and subsequent high-intensity endurance capacity. Int. J. Sport Nutr. Exerc. Metab. 2010, 20, 216-223.

24. Ferguson-Stegall, L.; McCleave, E.L.; Ding, Z.; Kammer, L.M.; Wang, B.; Doerner, P.G.; Liu, Y.; Ivy, J.L. The effect of a low carbohydrate beverage with added protein on cycling endurance performance in trained athletes. J. Strength Cond. Res. 2010, 24, 2577-2586.

25. Volianitis, S.; McConnell, A.K.; Koutedakis, Y.; Jones, D.A. The influence of prior activity upon inspiratory muscle strength in rowers and non-rowers. Int. J. Sports Med. 1999, 20, 542-547.

26. Johnson, M.A.; Gregson, I.R.; Mills, D.E.; Gonzalez, J.T.; Sharpe, G.R. Inspiratory muscle warm-up does not improve cycling time-trial performance. Eur. J. Appl. Physiol. 2014, 114, 1821-1830.

27. Chin, L.M.; Heigenhauser, G.J.; Paterson, D.H.; Kowalchuk, J.M. Effect of voluntary hyperventilation with supplemental $\mathrm{CO}_{2}$ on pulmonary $\mathrm{O}_{2}$ uptake and leg blood flow kinetics during moderate-intensity exercise. Exp. Physiol. 2013, 98, 1668-1682.

28. Fan, J.L.; Bourdillon, N.; Kayser, B. Effect of end-tidal $\mathrm{CO}_{2}$ clamping on cerebrovascular function, oxygenation, and performance during 15-km time trial cycling in severe normobaric hypoxia: The role of cerebral $\mathrm{O}_{2}$ delivery. Physiol. Rep. 2013, 1, doi:10.1002/phy2.66.

29. Enright, S.J.; Unnithan, V.B.; Heward, C.; Withnall, L.; Davies, D.H. Effect of high-intensity inspiratory muscle training on lung volumes, diaphragm thickness, and exercise capacity in subjects who are healthy. Phys. Ther. 2006, 86, 345-354.

30. Kilding, A.E.; Brown, S.; McConnell, A.K. Inspiratory muscle training improves 100 and $200 \mathrm{~m}$ swimming performance. Eur. J. Appl. Physiol. 2010, 108, 505-511.

31. Romer, L.M.; McConnell, A.K. Specificity and reversibility of inspiratory muscle training. Med. Sci. Sports Exerc. 2003, 35, 237-244. 
32. Jain, P.; Kavuru, M.S. A practical guide for peak expiratory flow monitoring in asthma patients. Clevel. Clin. J. Med. 1997, 64, 195-202.

(C) 2015 by the authors; licensee MDPI, Basel, Switzerland. This article is an open access article distributed under the terms and conditions of the Creative Commons Attribution license (http://creativecommons.org/licenses/by/4.0/). 